Article

\title{
Synthesis of Cellulose-2,3-bis(3,5-dimethylphenylcarbamate) in an Ionic Liquid and Its Chiral Separation Efficiency as Stationary Phase
}

\author{
Runqiang Liu ${ }^{1,2}$, Yijun Zhang ${ }^{2}$, Lianyang Bai ${ }^{1,3, *}$, Mingxian Huang ${ }^{2}$, Jun Chen ${ }^{2}$ \\ and Yuping Zhang ${ }^{2, *}$
}

1 College of Plant Protection, Hunan Agricultural University, Changsha 410128, China;

E-Mail: 1rq@hist.edu.cn

2 College of Chemistry and Chemical Engineering, Henan Institute of Science and Technology, Xinxiang 453003, China; E-Mails: zhangyijun@hist.edu.cn (Y.Z.);

huangmingxian@ hist.edu.cn (M.H.); junchen713@hist.edu.cn (J.C.)

3 Hunan Academy of Agricultural Sciences, Changsha 410125, China

* Authors to whom correspondence should be addressed;

E-Mails: blyang2006@163.com (L.B.); zhangyuping@hist.edu.cn (Y.Z.);

Tel.: +86-731-8469-2799 (L.B.); +86-373-3040-926 (Y.Z.);

Fax: +86-731-8469-1624 (L.B.); +86-373-3040-159 (Y.Z.).

Received: 11 February 2014; in revised form: 26 March 2014 / Accepted: 31 March 2014 /

Published: 11 April 2014

\begin{abstract}
A chiral selector of cellulose-2,3-bis(3,5-dimethylphenylcarbamate) (CBDMPC) was synthesized by reacting 3,5-dimethylphenyl isocyanate with microcrystalline cellulose dissolved in an ionic liquid of 1-allyl-3-methyl-imidazolium chloride (AMIMCl). The obtained chiral selector was effectively characterized by infrared spectroscopy, elemental analysis and ${ }^{1} \mathrm{H}$ NMR. The selector was reacted with 3-aminopropylsilanized silica gel and the CBDMPC bonded chiral stationary phase (CSP) was obtained. Chromatographic evaluation of the prepared CSPs was conducted by high performance liquid chromatographic (HPLC) and baseline separation of three typical fungicides including hexaconazole, metalaxyl and myclobutanil was achieved using n-hexane/isopropanol as the mobile phase with a flow rate $1.0 \mathrm{~mL} / \mathrm{min}$. Experimental results also showed that $\mathrm{AMIMCl}$ could be recycled easily and reused in the preparation of CSPs as an effective reaction media.
\end{abstract}


Keywords: 1-allyl-3-methyl-imidazolium chloride; synthesis; Cellulose-2,3-bis(3,5dimethylphenylcarbamate); high performance liquid chromatography; chiral stationary phase; enantioseparation

\section{Introduction}

Cellulose derivatives provide broad recognitions for a wide range of enantiomers, which makes them one of the most important chiral stationary phases (CSPs) in the field of separation sciences. To develop new application, synthesis methods of cellulose derivatives have received considerable attention in the field of enantioseparation [1,2]. However, the poor solubility of cellulose in common organic solvents and water has held back its functionality effectively. Okamoto et al. have ever developed some typical CSPs including coated amylose- and cellulose-derived CSPs, bonded amylose- and cellulose-derived CSPs [3-7]. Zhang group also reported the preparation of two kinds of CSPs by coating and bonding cellulose-tris (3,5-dimethylphenylcarbamate) on the surfaces of aminopropyl-functionalized silica gel, respectively [8]. The tritylation of cellulose derivatives in the synthesis of these CSPs is generally carried out through the reaction between its hydroxyl groups and reactive isocyanates in pyridine. After the activated cellulose is transferred to dry pyridine, a slurry is then formed that becomes homogenous as the reaction progresses. Generally, pyridine used as the reaction solvents has certain disadvantages, such as instability, high toxicity, difficulty in recovery and high cost, to a certain degree. Therefore, the development of environment-friendly, low-cost reaction solvents for synthesizing homogeneous derivatives of cellulose is of great significance for the preparation of high quality cellulose derivative products. Ionic liquid is an organic salt which is liquid at room temperature. It has attracted wide attention in the field of chemistry for its non-volatility, low melting point, adjustable structure and properties, and other advantages [9]. Recently, Granström et al. synthesized selectively protected 2,6-di-O-(4-methoxytrityl)cellulose in an ionic liquid of 1-allyl-3-methyl-imidazolium chloride (AMIMCl). It showed that AMIMCl not only functions as solvents for cellulose, but also be capable of increasing the reactivity [10]. Xu et al. synthesized cellulose dehydroabietate by the $O$-acylation reaction of cellulose with dehydroabietic acid chloride using ionic liquid 1-butyl-3-methylimidazolium bromide as a solvent [11]. These findings open a new field for investigating and developing the cellulose solvent system.

In the present study, cellulose-2,3-bis(3,5-dimethylphenylcarbamate) (CBDMPC) was synthesized in ionic liquid AMIMCl and the CBDMPC bonded chiral stationary phase (CSP) was obtained and evaluated.

\section{Results and Discussion}

\subsection{Synthesis and Characterization of CBDMPC}

The synthesis of CBDMPC includes tritylation, carbamation and the removal of methoxy-substituted trityl groups (Figure 1) [12]. In the present study, the microcrystalline cellulose was firstly dissolved in ionic liquid AMIMCl to form homogeneous solution; Secondly, the solution was treated with trityl 
chloride. Because of steric demands, trityl chloride reacts preferentially with the primary hydroxyl group at the $O-6$ position on the cellulose backbone rather than with either of the secondary hydroxyl groups at the $O-2 / 3$ positions [13]; Thirdly, the reaction solution was added 3,5-dimethylphenyl isocyanate to produce carbamate; Finally, the hydrolysis of the reaction solution in hydrochloride acid and methanol mixture afforded the titled compound. FTIR spectra of microcrystalline cellulose and CBDMPC were compared (Figure 2). A significant weaker - $\mathrm{OH}$ absorption peak appeared at $3415.33 \mathrm{~cm}^{-1}$ in the spectrum of CBDMPC. It indicated that most $-\mathrm{OH}$ groups of cellulose were replaced. The $\mathrm{C}-\mathrm{H}$ stretching vibration peak of $-\mathrm{CH}_{3}$ occurred at $2902.34 \mathrm{~cm}^{-1}$, the absorption peaks of the benzene ring were observed at $1617.98,1540.84$ and $1446.35 \mathrm{~cm}^{-1}$, and the absorption peak of the carbonyl group occurred at $1731.76 \mathrm{~cm}^{-1}$. The results demonstrate that the $-\mathrm{OH}$ groups on microcrystalline cellulose had reacted with 3,5-dimethylphenyl isocyanate. Preparation of CSPs was carried out by bonding CBDMPC on the surfaces of 3-aminopropyltriethoxysilane -modified silica gel (for detail, refer to experiment Section 3.3).

Figure 1. Synthesis of cellulose-2,3-bis(3,5-dimethylphenylcarbamate) (CBDMPC).
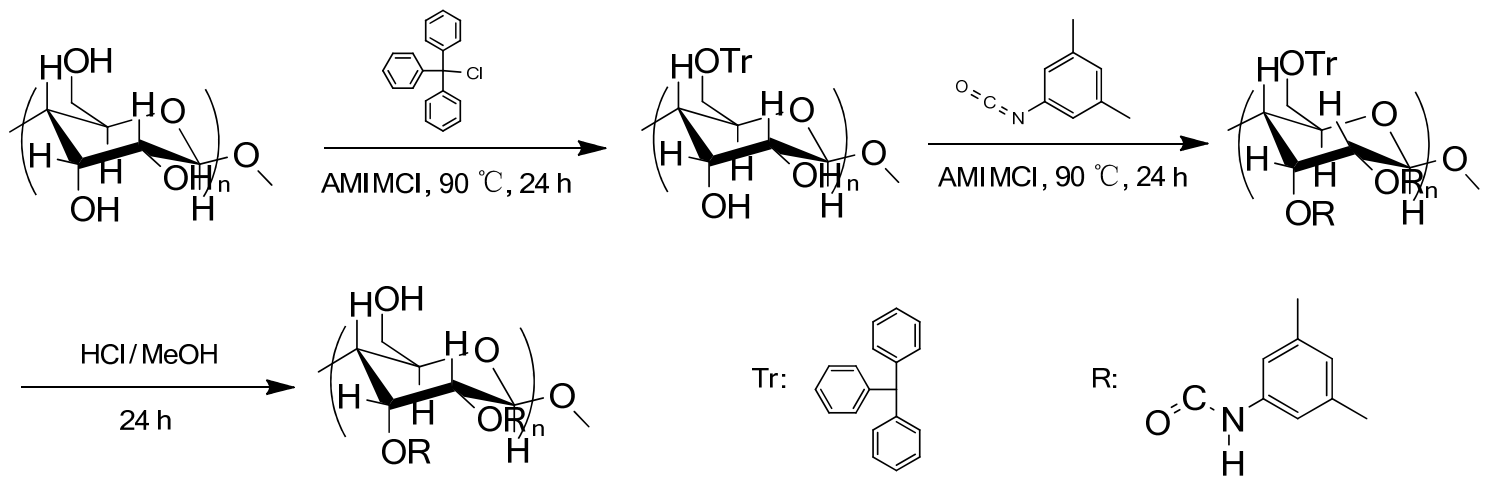

Figure 2. IR spectra of microcrystalline cellulose and CBDMPC. (1) microcrystalline cellulose; (2) CBDMPC.

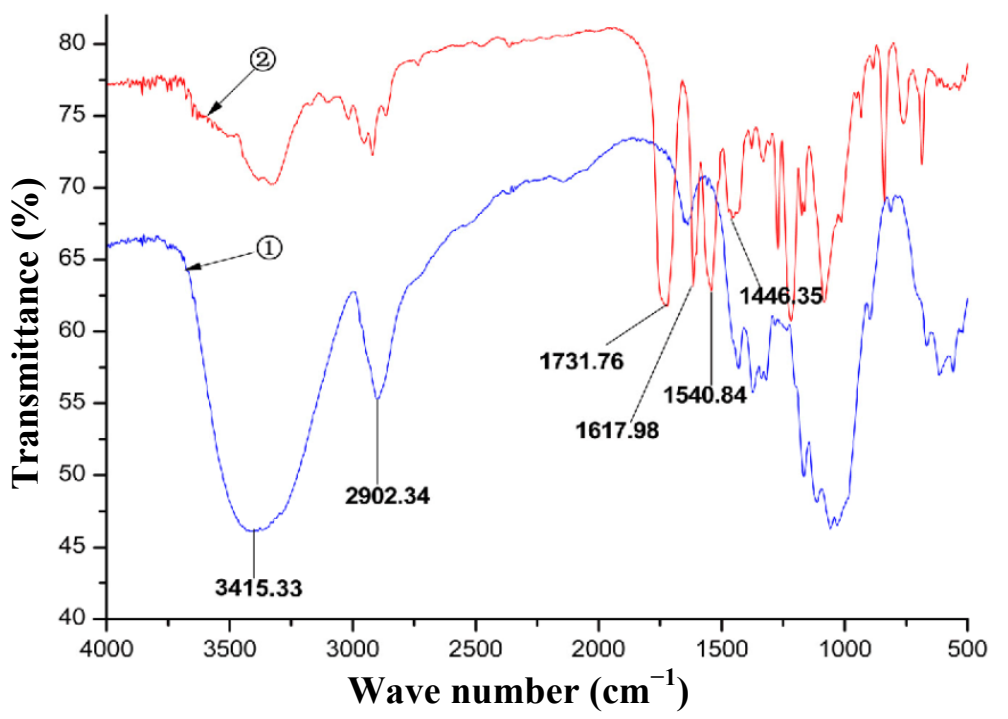

The obtained CBDMPC was also determined by the ${ }^{1} \mathrm{H}$ NMR (400 MHz, $50{ }^{\circ} \mathrm{C}$, DMSO- $d_{6}$ ) and the chemical shifts $(\delta, \mathrm{ppm})$ were assigned as follows: 8.6-9.0 $(\mathrm{N}-\mathrm{H}), 6.3-7.0(\mathrm{Ar}-\mathrm{H}), 3.6-5.0(\mathrm{Glucose}-\mathrm{H})$ 
and 2.0-2.1 $\left(\mathrm{Ar}-\mathrm{CH}_{3}\right)$. Moreover, according to the molecular formula $\left(\mathrm{C}_{24} \mathrm{H}_{28} \mathrm{O}_{7} \mathrm{~N}_{2}\right)_{\mathrm{n}}$ of CBDMPC, the theoretical percentage of each element could be calculated as follows: $\mathrm{C} 63.18 \%, \mathrm{H} 6.18 \%$ and $\mathrm{N} 6.14 \%$. In contrast, the measured values for each element by elemental analysis were $\mathrm{C} 64.57 \%$, $\mathrm{H}$ 6.26\% and $\mathrm{N} 6.56 \%$, respectively. Little difference between the theoretical and measured values for each element further demonstrated that cellulose-2,3-bis(3,5-dimethylphenylcarbamate) was successfully synthesized.

\subsection{Enantioseparation of Three Fungicides Using the Prepared Chiral Column}

In order to investigate the ability of chiral separation of CBDMPC-bonded CSPs, three typical fungicides including hexaconazole, metalaxyl and myclobutanil were selected for the evaluation in HPLC. Chromatographic conditions were selected with a flow rate of $1.0 \mathrm{~mL} / \mathrm{min}$ and a column temperature of $25^{\circ} \mathrm{C}$. The UV detection wavelength was set at $230 \mathrm{~nm}$ for hexaconazole, $214 \mathrm{~nm}$ for metalaxyl, and $210 \mathrm{~nm}$ for myclobutanil, respectively. The dead time was determined through the injection of 1,3,5-tri-tert-butylbenzene. Some chromatographic parameters including the resolution $\left(R_{\mathrm{s}}\right)$, capacity factor $\left(k^{\prime}\right)$, separation factor $(\alpha)$ for three chiral fungicides were listed in Table 1 . It was easily understood that the values of $k^{\prime}, \alpha$ and $R_{\mathrm{s}}$ decreased with the increase of isopropanol from $5 \%-30 \%$ in the mobile phases. This is because that hydrogen bond force of the sample molecules in the mobile phase increased with the decrease in isopropanol content, which decreased the sorption and desorption rates of the sample molecules in the mobile phase, thus increasing the separation power. The optimal chromatograms of three chiral fungicides are typically shown in Figure 3.

Table 1. Effect of isopropanol content on enantioseparation of three fungicides.

\begin{tabular}{cccccc}
\hline Compound & $\boldsymbol{n}$-Hexane/Isopropanol $(\boldsymbol{v} / \boldsymbol{v})$ & $\boldsymbol{k}_{\mathbf{1}}^{\prime}$ & $\boldsymbol{k}_{\mathbf{2}}^{\prime}$ & $\boldsymbol{\alpha}$ & $\boldsymbol{R}_{\mathbf{s}}$ \\
\hline \multirow{4}{*}{ Hexaconazole } & $70 / 30$ & 2.81 & 7.35 & 2.62 & 5.23 \\
& $75 / 25$ & 3.68 & 9.70 & 2.64 & 5.50 \\
& $80 / 20$ & 4.84 & 13.18 & 2.73 & 6.05 \\
& $85 / 15$ & 7.52 & 21.14 & 2.81 & 9.30 \\
& $90 / 10$ & 12.10 & 35.51 & 2.94 & 11.23 \\
\hline \multirow{4}{*}{ Metalaxyl } & $75 / 25$ & 1.92 & 3.37 & 1.76 & 2.64 \\
& $80 / 20$ & 2.58 & 4.73 & 1.83 & 3.62 \\
& $85 / 15$ & 3.27 & 6.09 & 1.86 & 3.74 \\
& $90 / 10$ & 4.84 & 9.45 & 1.95 & 3.89 \\
& $95 / 5$ & 9.59 & 20.25 & 2.11 & 4.37 \\
& $98 / 2$ & 22.02 & 49.12 & 2.23 & 11.74 \\
\hline \multirow{3}{*}{ Myclobutanil } & $70 / 30$ & 2.85 & 7.28 & 2.55 & 5.01 \\
& $75 / 25$ & 3.69 & 9.50 & 2.57 & 5.29 \\
& $80 / 20$ & 5.29 & 13.97 & 2.64 & 6.08 \\
& $85 / 15$ & 6.86 & 19.84 & 2.89 & 6.39 \\
& $90 / 10$ & 12.01 & 34.89 & 2.91 & 7.09 \\
\hline
\end{tabular}

Compared with the coated CSPs, the bonded CSPs may enhance the chiral separations due to their more choices of mobile phases. Here, in order to examine the stability of the self-made chiral column, about $1 \%-5 \%(v / v)$ THF and $\mathrm{CHCl}_{3}$ as a polar modifier were added to the mobile phases 
( $n$-hexane/isopropanol), respectively. After long runs of chromatographic operation with a flow rate of $2.0 \mathrm{~mL} / \mathrm{min}$ at $35^{\circ} \mathrm{C}$, no abnormal phenomena, such as reduced column efficiency for the selected fungicides and unstable baseline, was observed, demonstrating CBDMPC were bonded on the silica gel tightly.

Figure 3. Optimal chromatograms of three fungicides and their molecular structures. Peak identification: 1 hexaconazole; 2 Metalaxyl; 3 Myclobutanil. Chromatographic conditions: Enantioseparation of hexaconazole and myclobutanil was conducted using $n$-hexane/isopropanol $(70 / 30, v / v)$ as the mobile phases at a flow rate of $1.0 \mathrm{~mL} / \mathrm{min}$, whilst enantioseparation of metalaxyl was conducted using $n$-hexane/isopropanol $(75 / 25, v / v)$ as the mobile phases at a flow rate of $1.0 \mathrm{~mL} / \mathrm{min}$.
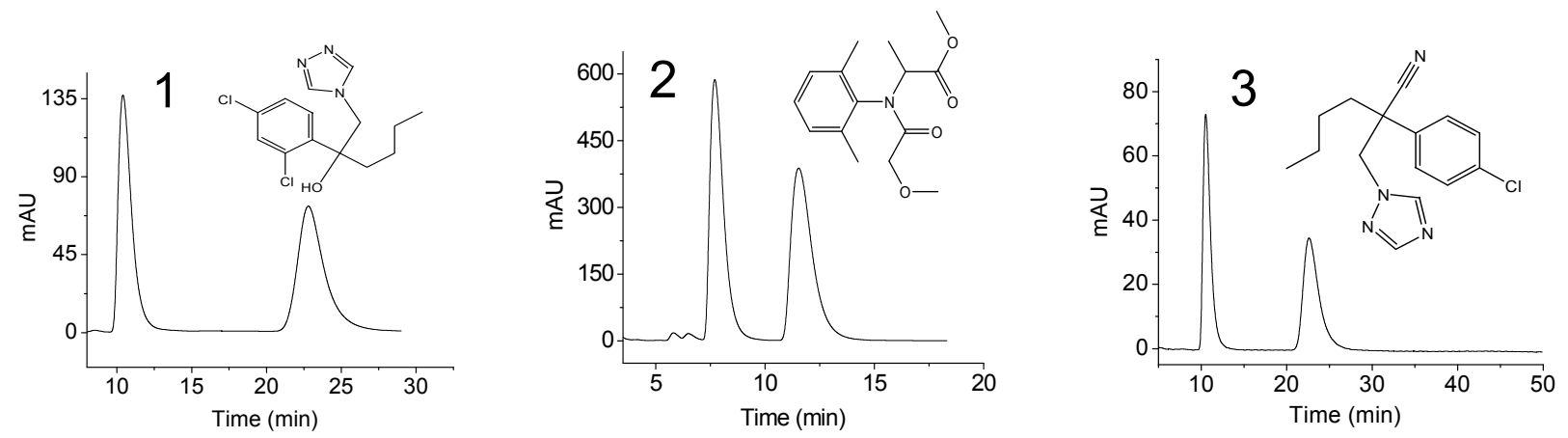

\subsection{Recycling and Characterization of the AMIMCl}

In view of environmental conservation and economics of the process, the solvent ionic liquid should be recycled and reused. In our study, at the end of carbamation of cellulose, the reaction mixture was poured into methanol, the CDMPC was precipitated and washed with methanol, and the filtrate was simply evaporated, giving a clean sample of AMIMCl, which was confirmed by IR and MS Spectroscopy. The fresh ionic liquid and recovered ionic liquid have almost the same spectral shapes, demonstrating that the purity of recovered ionic liquid is as high as that of the fresh ionic liquid (for detail, refer to supplementary).

\section{Experimental Section}

\subsection{Chemicals and Equipments}

AMIMCl was purchased from Shanghai Cheng Jie Chemical Co., Ltd. (Shanghai, China). Spherical silica gel (Kromasil, $5 \mu \mathrm{m}$, pore size $10 \mathrm{~nm}$ ) was purchased from Akzo Noble (Nacka, Sweden). Microcrystalline cellulose (Avicel, $D P=200$ ), 3-aminopropyltriethoxysilane (KH-550), hexamethylene diisocyanate, and 3,5-dimethylphenyl isocyanate were purchased from Aladdin Chemistry Co., Ltd. (Shanghai, China). Hexaconazole standard sample (95\%) and metalaxyl standard sample (98\%) were provided by Hunan Institute for Food and Drug Control (Changsha, China). Myclobutanil standard sample (98\%) was provided by Hunan Research Institute of Chemical Industry (Changsha, China). The $n$-hexane, isopropanol, tetrahydrofuran (THF) and trichloromethane used for mobile phase were HPLC 
grade, and purchased from Tianjin Chemical Reagent Co., Inc. (Tianjin, China). All mobile phases were filtrated through a $0.45 \mu \mathrm{m}$ filter membrane and degassed by ultrasonication prior to use.

Chromatographic measurements were made on a Agilent 1100 HPLC system (Agilent Technologies, Inc., Walbronn, Germany) equipped with a quaternary pump, a vacuum degasser module, a Rheodyne $7725 \mathrm{i}$ injector with a $20 \mu \mathrm{L}$ sample loop, a temperature controlled column compartment and a variable wavelength UV detector. Fourier transform infrared spectroscopy (FTIR) spectra were recorded in the range $400-4000 \mathrm{~cm}^{-1}$ with $4 \mathrm{~cm}^{-1}$ resolution with a BRUKER TENSOR27 system (Bruker Scientific Technology Co., Ltd., Karlsruhe, Germany). The $400 \mathrm{MHz}$ ${ }^{1}$ H NMR spectra were measured by a BRUKER ARX 400 spectrometer (Bruker Scientific Technology Co., Ltd., Karlsruhe, Germany).

\subsection{Synthesis of Cellulose-2,3-bis(3,5-dimethylphenylcarbamate)}

One gram of dry microcrystalline cellulose (degree of polymerization of 200) was dissolved in $20 \mathrm{~mL}$ of ionic liquid $\mathrm{AMIMCl}$ at $90{ }^{\circ} \mathrm{C}$, then $3.50 \mathrm{~g}$ of triphenylchloromethane was added into the solution. The solution was reacted at $90{ }^{\circ} \mathrm{C}$ for $24 \mathrm{~h}$ under vigorously stirring. $6.6 \mathrm{~mL}$ of 3,5-dimethylphenyl isocyanate was added into the solution, and reacted for another $48 \mathrm{~h}$ at $90{ }^{\circ} \mathrm{C}$. After reaction was completed, the mixture was cooled and poured into $400 \mathrm{~mL}$ of anhydrous methanol containing $3.3 \mathrm{~mL}$ of concentrated hydrochloric acid, and the mixture was stirred at room temperature for $24 \mathrm{~h}$. The white precipitate was separated by suction filtration and washed several times with large amounts of anhydrous methanol. The collected solid was dried under vacuum at $50{ }^{\circ} \mathrm{C}$ to obtain the CBDMPC with a yield of $85 \%$.

\subsection{Preparation of Bonded-Type Chiral Stationary Phase}

In brief, $8 \mathrm{~g}$ of spherical silica gel was transferred into a $150 \mathrm{~mL}$ three-necked flask, then dried in vacuum at $110{ }^{\circ} \mathrm{C}$ for $24 \mathrm{~h}$, cooled to room temperature, and added with $80 \mathrm{~mL}$ of anhydrous toluene and $16 \mathrm{~mL}$ of 3-aminopropyltriethoxysilane. The resulting mixture was allowed to react at reflux for $48 \mathrm{~h}$. The entire process was conducted under a nitrogen atmosphere. The mixture was filtered after cooling, and then washed with an appropriate amount of toluene, methanol, diethyl ether, petroleum ether. The product was dried in vacuum at $60{ }^{\circ} \mathrm{C}$ for $5 \mathrm{~h}$ to obtain white 3 -aminopropylsilanized silica gel [14].

Nought point eight three gram of CBDMPC was dissolved in $10 \mathrm{~mL}$ of THF, followed by the addition of $3.30 \mathrm{~g} 3$-aminopropylsilanized silica gel. The solution was dried for $8 \mathrm{~h}$ under vacuum at $60{ }^{\circ} \mathrm{C}$ so as to remove the solvent. Afterwards, $30 \mu \mathrm{L}$ of hexamethylene diisocyanate and $10 \mathrm{~mL}$ of anhydrous toluene were added and thoroughly mixed. The mixture was allowed to react at $100{ }^{\circ} \mathrm{C}$ for $24 \mathrm{~h}$, and then added with $4.5 \mathrm{~mL}$ of 3,5-dimethylphenyl isocyanate. The resulted mixture was allowed to react at reflux for another $48 \mathrm{~h}$ and then filtered to obtain a solid product. Cellulose derivatives were then regioselectively bonded to silica gel on their glucose units [14]. The product was subjected to Soxhlet extraction for $24 \mathrm{~h}$ using THF and methanol, respectively, and then dried in vacuum at $60{ }^{\circ} \mathrm{C}$ for $24 \mathrm{~h}$ to obtain the final CSP products. 


\subsection{Packing}

Using isopropanol as the slurry solvent and $n$-hexane/isopropanol $(90 / 10, v / v)$ solution as the displacement fluid, the prepared CSP was packed into a stainless-steel column $(250 \mathrm{~mm} \times 4.6 \mathrm{~mm})$ at a pressure of 7000 Psi.

\section{Conclusions}

In conclusion, the green solvent, ionic liquid $\mathrm{AMIMCl}$ can absolutely replace the traditional solvent pyridine in the synthesis of the chiral selector cellulose-2,3-bis(3,5-dimethylphenylcarbamate). Successful synthesis of the chiral selector was proved by three characterization methods: IR, ${ }^{1} \mathrm{H}$ NMR and elemental analysis. Chromatographic evaluation performed on the self-made chiral column in HPLC demonstrated that our developed method is effective for the green synthesis of CSPs.

\section{Acknowledgments}

The financial supports by the National Natural Science Foundation of China (Nos. 30070506, 30771436); key scientific and technological project of the Science and Technology Department of Henan province (No. 092102310227); natural science research project of the Education Department of Henan province (Nos. 2007150019, 2008A150012); are gratefully acknowledged.

\section{Author Contributions}

R.L. and Y.Z. designed the experiment under the supervision of L.B.; R.L., Y.Z. and Y.Z. performed the experiments and analyzed the data with the advice of M.H. and Y.Z.; R.L. and Y.Z. discussed the results; L.B. , Y.Z., J.C. and M.H. gave conceptual advice; R.L. wrote the manuscript; all authors commented on the manuscript at all stages.

\section{Conflicts of Interest}

The authors declare no conflict of interest.

\section{References}

1. Armstrong, D.W. The evolution of chiral stationary phases for liquid chromatography. J. Chin. Chem. Soc. 1998, 45, 581-590.

2. Lämmerhofer, M.; Svec, F.; Fréchet, J.M. Separation of enantiomers by capillary electrochromatography. J. Trends Anal. Chem. 2000, 19, 676-698.

3. Chankvetadze, B.; Yamamoto, C.; Okamoto, Y. Enantioseparation of selected chiral sulfoxides using polysaccharide-type chiral stationary phases and polar organic, polar aqueous-organic and normal-phase eluents. J. Chromatogr. A 2001, 922, 127-137.

4. Li, W.H.; Liu, C.H.; Tan, G.G.; Zhang, X.R.; Zhu, Z.Y.; Chai, Y.F. Molecular modeling study of chiral separation and recognition mechanism of $\beta$-adrenergic antagonists by capillary electrophoresis. Int. J. Mol. Sci. 2012, 13, 710-725. 
5. Okamoto, Y.; Kaida, Y. Resolution by high-performance liquid chromatography using polysaccharide carbamates and benzoates as chiral stationary phases. J. Chromatogr. A 1994, 666, 403-419.

6. Franco, P.; Minguillón, C.; Oliveros, L. Solvent versatility of bonded cellulose-derived chiral stationary phases for high-performance liquid chromatography and its consequences in column loadability. J. Chromatogr. A 1998, 793, 239-247.

7. Oliveros, L.; Senso, A.; Minguillon, C. Benzoates of cellulose bonded on silica gel: Chiral discrimination ability as high-performance liquid chromatographic chiral stationary phases. Chirality 1997, 9, 145-149.

8. Bai, L.Y.; Zhang, Y.P.; Deng, P.H.; Zhang, Y.J.; Chen, J. Enantioseparation of typical pesticides using cellulose carbamate stationary phases by capillary liquid chromatography. Asian J. Chem. 2012, 24, 4917-4922.

9. Zhang, J.M.; Wu, J.; Cao, Y.; Sang, S.M.; Zhang, J.; He, J.S. Synthesis of cellulose benzoates under homogeneous conditions in an ionic liquid. Cellulose 2009, 16, 299-308.

10. Granström, M.; Olszewska, A.; Makela, V.; Heikkinen, S.; Kilpelainen, I. A new protection group strategy for cellulose in an ionic liquid: Simultaneous protection of two sites to yield 2,6-di-O-substituted mono- $p$-methoxytrityl cellulose. Tetrahedron Lett. 2009, 50, 1744-1747.

11. Xu, X.T.; Duan, W.G.; Huang, M.; Li, G.H. Synthesis of cellulose dehydroabietate in ionic liquid [bmim] Br. Carbohydr. Res. 2011, 346, 2024-2027.

12. Chankvetadze, B.; Ikai, T.; Yamamoto, C.; Okamoto, Y. High-performance liquid chromatographic enantioseparations on monolithic silica columns containing a covalently attached 3,5-dimethylphenylcarbamate derivative of cellulose. J. Chromatogr. A 2004, 1042, 55-60.

13. Yashima E.; Fukaya, H,; Okamoto, Y. 3,5-Dimethylphenylcarbamates of cellulose and amylase regioselectively bonded to silica gel as chiral stationary phases for high-performance liquid chromatography. J. Chromatogr. A 1994, 677, 11-19.

14. Ahmed, A.Y.; Mangelings, D.; Heyden, Y.V. Chiral separations in normal-phase liquid chromatography: Enantioselectivity of recently commercialized polysaccharide-based selectors. Part II. Optimization of enantioselectivity. J. Pharm. Biomed. Anal. 2011, 56, 521-537.

(C) 2014 by the authors; licensee MDPI, Basel, Switzerland. This article is an open access article distributed under the terms and conditions of the Creative Commons Attribution license (http://creativecommons.org/licenses/by/3.0/). 